\title{
Effect of Blood Group on Ultrahigh Frequency Auditory Sensitivity
}

\author{
Prashanth Prabhu ${ }^{1}$ Akhila Chandrashekhar ${ }^{1}$ Janani Cariappa ${ }^{1}$ Nayanika Ghosh ${ }^{1}$ \\ ${ }^{1}$ Department of Audiology, All India Institute of Speech and Hearing, \\ Mysore, Karnataka, India \\ Int Arch Otorhinolaryngol 2018;22:364-367. \\ Address for correspondence Prashanth Prabhu, MSc, Department of \\ Audiology, All India Institute of Speech and Hearing, Naimisham \\ Campus Manasagangothri, Mysore, Karnataka 570006, India \\ (e-mail: prashanth.audio@gmail.com).
}

\begin{abstract}
Introduction Individuals with blood group $\mathrm{O}$ are reported to have reduced otoacoustic emissions (OAEs) compared with individuals with different blood groups. Objective The present study attempted to determine if the blood group has any effect on high-frequency auditory sensitivity using ultrahigh-frequency audiometry and ultrahigh-frequency distortion product otoacoustic emissions (DPOAEs).

Methods High-frequency thresholds and high-frequency DPOAEs were measured in 60 individuals with normal hearing and different blood groups.

Results The results of the study showed that there was a significant reduction in

Keywords

- noise-induced hearing loss

- ABO blood group system

- audiometry DPOAE amplitude for individuals with blood group $\mathrm{O}$ compared with individuals with other blood groups. However, there was no significant difference in ultrahighfrequency thresholds across the blood groups.

Conclusion This reduction in OAE amplitude may be attributed to a lower number of healthy outer hair cells in individuals with blood group O. Further studies on larger groups of individuals are essential for a better generalization of the results.
\end{abstract}

\section{Introduction}

The susceptibility to a high level of noise is reported to vary across different individuals. ${ }^{1}$ There are several factors such as the amount of melanin, race, cholesterol level, blood pressure etc. that are reported to affect susceptibility to noise. ${ }^{2}$ Recently, Chow, McPherson and Fuente ${ }^{3}$ reported that individuals with blood group $\mathrm{O}$ are relatively more susceptible to noise-induced hearing loss (NIHL). To confirm their hypothesis, measuring ultrahigh-frequency auditory sensitivity in individuals with different blood groups would be beneficial. Ultrahigh-frequency audiometry is also used extensively to monitor the hearing sensitivity, as it is used to assess the early auditory involvement. ${ }^{4}$ It is well-reported that monitoring ultrahigh frequencies is useful in the early detection of NIHL and ototoxicity. ${ }^{4-6}$ In addition, the evaluation of otoacoustic emissions (OAEs), especially at high frequencies, is one of the most sensitive tests of hearing. 4,7

received

June 14, 2017

accepted

October 10, 2017

published online

December 15, 2017
Previous studies have reported that genetic differences can influence the susceptibility to certain disorders in individuals with different blood groups. ${ }^{8}$ Individuals with blood group 0 are particularly reported to be more susceptible to NIHL. ${ }^{3,9}$ Chow, McPherson and Fuente ${ }^{3}$ assessed the presence of spontaneous otoacoustic emissions (SOAEs), the amplitude of transient-evoked otoacoustic emissions (TEOAEs) and distortion product otoacoustic emissions (DPOAEs) across different blood groups in 60 adult females with normal hearing. They reported that the SOAEs were mostly absent in individuals with blood group 0 , and the amplitude of their DPOAEs was significantly reduced compared with other blood groups. They hypothesized that this difference could be related to a higher susceptibility to NIHL in individuals with blood group 0 compared with other blood groups. However, they suggested that this hypothesis should be confirmed by further studies assessing the effect of blood group on OAEs. It is reported in the literature that ultrahigh-frequency audiometry and ultrahigh-

Copyright @ 2018 by Thieme Revinter

Publicações Ltda, Rio de Janeiro, Brazil
License terms

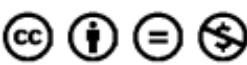


frequency DPOAEs are early indicators of NIHL. ${ }^{4,10,11}$ However, a recent systematic review suggests that high-frequency audiometry may not be an optimal tool to identify NIHL. ${ }^{12}$ The present study attempted to analyze the effect of blood group on ultrahigh-frequency thresholds and ultrahigh-frequency DPOAEs in young adult females with normal hearing. The study attempted to understand if there are differences in ultrahigh-frequency thresholds and ultrahigh-frequency DPOAE amplitudes in individuals with different blood groups.

\section{Materials and Methods}

\section{Participants}

A total of 60 adults females with normal hearing, aged between 17 years and 25 years (mean age: 19.2 years; standard deviation [SD]: 5.6) participated in the study, and 15 participants were included in each blood group ( $A, B, A B$ and $O)$. The mean age of the participants with $A, B, A B$ and $O$ blood groups was of 18.25 years (SD: 2.7), 19.73 years (SD: 4.6), 18.91 years (SD: 3.9) and 19.21 years (SD: 5.1) respectively. The rhesus (Rh) was positive in all of the participants of the study. Only female participants were considered because the amplitude of the DPOAEs is reported to vary across gender. ${ }^{13}$ The participants with no significant otological history, noise exposure, history of smoking, intake of ototoxic drugs, diabetes and familial hearing loss were included in the study. The participants were not exposed to any loud noise 24 hours prior to the test. All of the participants had audiometric thresholds $<15 \mathrm{~dB}$ HL from $250 \mathrm{~Hz}$ to $8 \mathrm{kHz}$. The immittance evaluation showed normal middle ear function in all participants.

\section{Procedure}

A two-channel diagnostic audiometer Inventis Piano (Inventis, Padova, Italy) was used to obtain air conduction and bone conduction pure tone thresholds and speech identification scores. Speech identification scores using headphones (Telephonics, Farmingdale, NY, USA) were obtained for phonemicallybalanced words developed for adults in Kannada by Yathiraj and Vijayalakshmi. ${ }^{14}$ Recorded word lists were routed from a personal computer through the two-channel diagnostic audiometer at $40 \mathrm{dBSL}$ (re: speech recognition threshold). A Tympstar (GrasonStadler, Inc., Eden Prairie, MN, US) immittance meter was used for immittance testing. The better ear of the participant was tested to obtain tympanogram and acoustic reflexes for a probe tone frequency of $226 \mathrm{~Hz}$. The acoustic reflexes were measured using $500 \mathrm{~Hz}, 1,000 \mathrm{~Hz}, 2,000 \mathrm{~Hz}$, and $4000 \mathrm{~Hz}$ pure tones, presented to both ipsilateral and contralateral ears.

High-frequency thresholds for both ears were determined using a calibrated audiometer with HDA-200 headphones (Sennheiser, Wedemark, Germany) at the frequencies of $9 \mathrm{kHz}, 10 \mathrm{kHz}, 11.25 \mathrm{kHz}, 12.5 \mathrm{kHz}, 14 \mathrm{kHz}$, and $16 \mathrm{kHz}$. The thresholds were obtained using a modified version of the Hughson and Westlake method. ${ }^{15}$ High-frequency DPOAE measurements were recorded for both ears using the Mimosa Acoustics (Champaign, IL, US) OAEs equipment in a soundtreated room. The appropriate probe fitting was ensured, and the stimulus was calibrated before recording the OAEs. Highfrequency DPOAEs were recorded at the $\mathrm{f} 2 / \mathrm{f} 1$ ratio of 1.22 with the intensity of f1 (L1) at $65 \mathrm{~dB}$ SPL and the intensity of $\mathrm{f} 2$ (L2) at $55 \mathrm{~dB}$ SPL. High-frequency DPOAEs were measured across the frequencies of $9 \mathrm{kHz}, 10.25 \mathrm{kHz}, 11.5 \mathrm{kHz}, 12.5 \mathrm{kHz}, 14 \mathrm{kHz}$, and $16 \mathrm{kHz}$.

\section{Ethical Considerations}

In the present study, all of the testing procedures were performed using a non-invasive technique and adhering to the conditions of the ethical approval committee of the institute. All of the test procedures were explained to the patients and their family members before testing, and informed consent was taken from all patients or their family members for their participation in the study.

\section{Results}

The results of the study showed that the ultrahigh-frequency thresholds were similar across the different blood groups. The means and SDs of the thresholds across all four blood groups are shown in - Fig. 1. The normality of the data distribution was assessed using the Shapiro-Wilk test of normality. The results of the Shapiro-Wilk test showed that the data was normally distributed $(p>0.05)$. Hence, parametric inferential statistics were administered on the data. Since there were multiple dependent variables, the multiple analyses of variance (MANOVA) test was administered. The results of the MANOVA considering ultrahighfrequencies as dependent variables showed that there was no significant difference $(p>0.05)$ in ultrahigh-frequency thresholds across blood groups.

We also found that the amplitude of ultrahigh-frequency DPOAEs was reduced for individuals with blood group 0 when compared with other blood groups. The means and SDs of DPOAE amplitude across all blood groups are shown in - Fig. 2. The results of the MANOVA also showed that there was a significant difference $(p<0.01)$ in the amplitude of ultrahigh-frequency DPOAEs. Furthermore, a post hoc analysis of the data showed that the high-frequency DPOAEs were significantly reduced $(p<0.01)$ at all frequencies for blood group 0 compared with other blood groups. There was no significant difference $(p>0.05)$ in the amplitude of DPOAEs between blood groups A, B and AB.

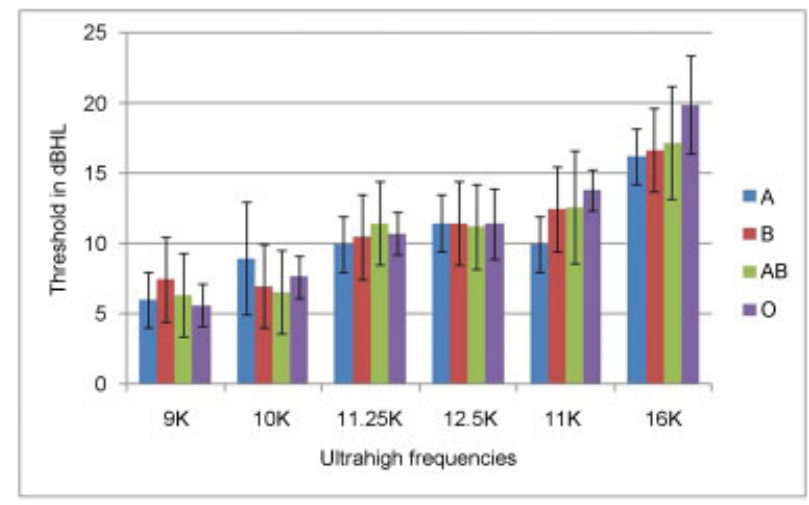

Fig. 1 Mean and standard deviation of ultrahigh-frequency thresholds across four blood groups. 


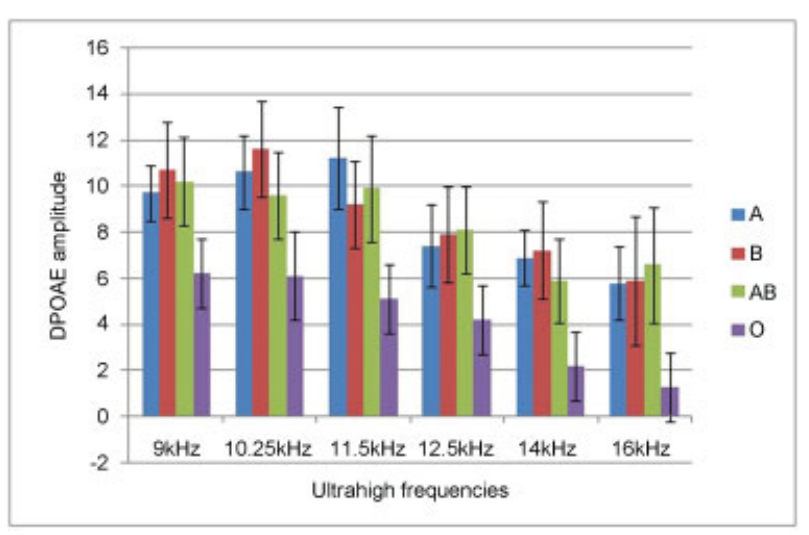

Fig. 2 Mean and standard deviation of ultrahigh frequency DPOAEs across four blood groups.

The amplitudes of ultrahigh-frequency DPOAEs and ultrahigh-frequency thresholds were averaged, and the mean differences across blood groups were also analyzed. Since the MANOVA showed a significant difference, separate one-way analysis of variance (ANOVA) was administered on the data. The results of the one-way ANOVA showed no significant difference $(p>0.05)$ in average ultrahigh-frequency thresholds across blood groups. The ANOVA results also showed a significant difference $(p<0.01)$ in the averaged ultrahigh-frequency DPOAEs across blood groups. The post hoc analysis showed that the average DPOAE amplitude was significantly lower $(p<0.01)$ for blood group 0 compared with other blood groups.

\section{Discussion}

The results of the study showed that there was a reduction in DPOAE amplitude for individuals with blood group 0 compared with their counterparts with different blood groups. However, no changes in ultrahigh-frequency thresholds were noted across the different blood groups. Chow, McPherson and Fuente $^{3}$ also reported that individuals with blood group $O$ had lesser occurrence of SOAEs and reduced TEOAE and DPOAE amplitudes compared with the other blood groups. The reduction in amplitude of ultrahigh-frequency DPOAEs in blood group $\mathrm{O}$ indicates a possible reduction in outer hair cell $(\mathrm{OHC})$ function. Thus, differences in the number of healthy OHCs may be associated with the reduction in amplitude of DPOAEs. ${ }^{16,17}$ The results of the present study are in consensus with reports by Chow, McPherson and Fuente, ${ }^{3}$ who also reported reduced OAEs in individuals with blood group 0 .

Previous studies have reported that genetic differences can influence the susceptibility to certain disorders in individuals with different blood groups. ${ }^{8}$ Thus, the lower ultrahigh-frequency OAEs observed in individuals with blood group 0 suggest that they might be congenitally predisposed to have fewer OHCs. The results of the study showed that there are no significant differences in ultrahigh-frequency thresholds across the blood groups. This suggests that the loss of OHCs in individuals with blood group $\mathrm{O}$ may not be large enough to cause an elevation in threshold. The difference in blood group could just be a genetic predisposition. However, this is pure speculation, and it should be confirmed by other objective studies. Other factors like larger ear canal volume or stronger efferent system could have reduced OAE amplitude, and this needs to be further explored. Thus, the present study is just a preliminary evaluation of the difference in ultrahigh-frequency thresholds and ultrahigh-frequency OAEs in individuals with different blood groups. However, further studies are essential to explore the possible reasons for these differences in individuals with different blood groups.

\section{Conclusions}

The present study attempted to determine the effect of the type of blood group on high-frequency auditory sensitivity using ultrahigh-frequency audiometry and ultrahigh frequency DPOAEs. The results of the study showed that there was a significant reduction in DPOAE amplitude for individuals with blood group $\mathrm{O}$ compared with individuals with other blood groups. However, there was no significant difference in ultrahigh-frequency threshold across the blood groups. Thus, one could speculate that the reduction in OAE amplitude can be attributed to a congenital lower number of healthy OHCs. Further studies on larger groups of individuals are essential to generalize the results.

\section{Conflicts of Interest}

The authors report no conflicts of interest. The authors alone are responsible for the content and writing of the paper.

\section{References}

1 Plontke S, Zenner H-P. Current aspects of hearing loss from occupational and leisure noise. GMS Curr Top Otorhinolaryngol Head Neck Surg 2004;3:Doc06

2 Henderson D, Subramaniam M, Boettcher FA. Individual susceptibility to noise-induced hearing loss: an old topic revisited. Ear Hear 1993;14(03):152-168

3 Chow KT, McPherson B, Fuente A. Otoacoustic emissions in young adults: Effects of blood group. Hear Res 2016;333:194-200

4 Mehrparvar AH, Mirmohammadi SJ, Ghoreyshi A, Mollasadeghi A, Loukzadeh Z. High-frequency audiometry: a means for early diagnosis of noise-induced hearing loss. Noise Health 2011;13 (55):402-406

5 Singh Chauhan R, Saxena RK, Varshey S. The role of ultrahighfrequency audiometry in the early detection of systemic druginduced hearing loss. Ear Nose Throat J 2011;90(05):218-222

6 van der Hulst RJ, Boeschoten EW, Nielsen FW, Struijk DG, Dreschler WD, Tange RA. Ototoxicity monitoring with ultrahigh frequency audiometry in peritoneal dialysis patients treated with vancomycin or gentamicin. ORL J Otorhinolaryngol Relat Spec 1991;53(01):19-22

7 Kemp DT, Ryan S, Bray P. A guide to the effective use of otoacoustic emissions. Ear Hear 1990;11(02):93-105

8 Sircar S. Principles of Medical Physiology. New York: Thieme; 2008:161-162 p

9 Lashley FR. Clinical Genetics in Nursing Practice. 3rd ed. New York: Springer; 2005:387-400 p

10 Somma G, Pietroiusti A, Magrini A, et al. Extended high-frequency audiometry and noise induced hearing loss in cement workers. Am J Ind Med 2008;51(06):452-462 
11 Korres GS, Balatsouras DG, Tzagaroulakis A, Kandiloros D, Ferekidis E. Extended high-frequency audiometry in subjects exposed to occupational noise. B-ENT 2008;4(03):147-155

12 Antonioli CAS, Momensohn-Santos TM, Benaglia TAS. High-frequency Audiometry Hearing on Monitoring of Individuals Exposed to Occupational Noise: A Systematic Review. Int Arch Otorhinolaryngol 2016;20(03):281-289

13 Fernandes LC, Santos TMM. Tinnitus and normal hearing: a study on the transient otoacoustic emissions suppression. Rev Bras Otorrinolaringol (Engl Ed) 2016;75(03):414-419
14 Yathiraj A, Vijayalakshmi CS. Phonemically balanced wordlist in Kannada. University of Mysore; 2005

15 Carhart R, Jerger JF. Preferred method for clinical determination of pure-tone thresholds. J Speech Hear Disord 1959;24:330-345

16 Kemp DT. Otoacoustic emissions, their origin in cochlear function, and use. Br Med Bull 2002;63:223-241

17 Lonsbury-Martin BL, Martin GK. Distortion-product otoacoustic emissions in 421 populations with normal hearing sensitivity. In: Robinette MS, Glattke TJ. Otoacoustic Emissions: Clinical Applications. 3rd ed. New York: Thieme; 2007:107-130 